International Mathematical Forum, 1, 2006, no. 19, 933-946

\title{
Nonlinear Neutral Integrodifferential Equations on Unbounded Intervals
}

\author{
Giuseppe Marino and Paolamaria Pietramala
}

Dipartimento di Matematica, Universita della Calabria

87036 Arcavacata di Rende (Cs), Italy

gmarino@unical.it, pietramala@unical.it

Hong-Kun $\mathrm{Xu}^{1}$

Department of Mathematics, University of Durban-Westville

Private Bag X54001, Durban 4000, South Africa

hkxu@pixie.udw.ac.za

\begin{abstract}
In this paper we prove the existence of solutions for a boundary value nonlinear neutral integrodifferential problem in $\mathbf{R}^{n}$ defined on an unbounded interval. The result is obtained by using the Schaefer fixed point theorem and by using a recent result [4] on compactness of a continuous operator $K: \mathcal{B C}\left(I, \mathbf{R}^{n}\right) \rightarrow \mathcal{B C}\left(I, \mathbf{R}^{n}\right)$; here $\mathcal{B C}\left(I, \mathbf{R}^{n}\right)$ is the Banach space of continuous functions from the (possibly) unbounded interval $I \subset \mathbf{R}$ into $\mathbf{R}^{n}$. As corollary we obtain a result of existence of solutions for a Cauchy problem for nonlinear neutral integrodifferential equations.
\end{abstract}

Keywords: Nonlinear neutral integrodifferential equation, existence of solution, Schaefer's fixed point theorem, compactness, boundary value problem, unbounded interval

Mathematics Subject Classification: 90C30, 90C33

\section{Introduction}

We are concerned with the existence of strong solutions of nonlinear integrodifferential equations of the form

$\frac{d}{d t}\left[x(t)-g\left(t, x_{t}\right)\right]=A(t) x(t)+f\left(t, x_{t}, \int_{0}^{t} h\left(t, s, x_{s}\right) d s\right), \quad t \in[0,+\infty)$

\footnotetext{
${ }^{1}$ Supported in part by the South African National Research Foundation.
} 
with the boundary condition

$$
L(x)=H(x)
$$

where

- $A(t)$ is a linear operator on $\mathbf{R}^{n}$ for $t \geq 0$;

- $h:[0,+\infty) \times[0,+\infty) \times \mathcal{C}\left([-r, 0] ; \mathbf{R}^{n}\right) \rightarrow \mathbf{R}^{n}, f:[0,+\infty) \times \mathcal{C}\left([-r, 0] ; \mathbf{R}^{n}\right) \times$ $\mathbf{R}^{n} \rightarrow \mathbf{R}^{n}$, and $g:[0,+\infty) \times \mathcal{C}\left([-r, 0] ; \mathbf{R}^{n}\right) \rightarrow \mathbf{R}^{n}$ are continuous functions;

- $\mathcal{C}\left([-r, 0] ; \mathbf{R}^{n}\right)$ is the Banach space of all continuous functions $y:[-r, 0] \rightarrow$ $\mathbf{R}^{n}$ endowed with the norm $\|y\|_{\infty}:=\max \{|y(\theta)|:-r \leq \theta \leq 0\}$;

- $\mathcal{B C}\left([-r,+\infty) ; \mathbf{R}^{n}\right)$ is the Banach space of all bounded continuous functions $y:[-r,+\infty) \rightarrow \mathbf{R}^{n}$ endowed with the norm $\|y\|_{\infty}:=\sup \{|y(t)|:$ $-r \leq t \leq+\infty\}$

- for each $y \in \mathcal{B C}\left([-r,+\infty) ; \mathbf{R}^{n}\right)$, we have $y_{t} \in \mathcal{C}\left([-r, 0] ; \mathbf{R}^{n}\right)$ for $t \geq 0$, where $y_{t}(\theta):=y(t+\theta)$ for $\theta \in[-r, 0]$;

- $L: \mathcal{B C}\left([-r,+\infty) ; \mathbf{R}^{n}\right) \rightarrow \mathcal{C}\left([-r, 0] ; \mathbf{R}^{n}\right)$ is a bounded linear operator;

- $H: \mathcal{B C}\left([-r,+\infty) ; \mathbf{R}^{n}\right) \rightarrow \mathcal{C}\left([-r, 0] ; \mathbf{R}^{n}\right)$ is a continuous bounded (in general, nonlinear) operator.

The theory of neutral delay differential equations has extensively been studied in the literature (see $[1,3,5,7]$ and the references therein). In particular, Benchohra, Henderson, and Ntouyas [1] recently obtained existence results for impulsive neutral functional differential equations in Banach spaces on finite intervals.

In all these cited papers, the equation (1) is studied in the case where $A(t)=A$ is time-independent and the time $t$ interval is a bounded interval $[0, b]$; moreover, the boundary value condition is always a Cauchy condition of the form

$$
x_{0}=\varphi \quad \text { on }[-r, 0] \text {, with } \varphi \in \mathcal{C}\left([-r, 0] ; \mathbf{R}^{n}\right) \text { fixed }
$$

We will obtain the existence of solutions from which such a Cauchy problem is a simple corollary to our main result with the general boundary value condition (2). It seems to us that not many existence results have so far been established to solve the general problem (1)-(2). Our main result, in particular, extends the main result (Theorem 3.1, p. 96) of Dauer and Balachandran [3] in twofold: Our operator $A(t)$ is time-dependent and the interval on which equation (1) is considered is infinite. Furthermore, our hypotheses are simpler than those in Dauer and Balachandran [3]. 


\section{Notation and Preliminary Results}

We use the following notation:

- $\mathbf{R}^{n}$, the space of real $n$-vectors $y$ with norm $|y|$ (not necessarily the Euclidean norm).

- $\mathcal{A}$, the algebra of real $n \times n$ matrices $M$ with norm $|M|=\sup \{|M y|$ : $|y|=1\}$.

- $t \mapsto A(t)$, a function from $[0,+\infty)$ into $\mathcal{A}$.

- $\|x\|_{\infty}=\sup \{|x(t)|: t \in[0,+\infty)\}$, the $\sup$ norm of a function $x \in$ $\mathcal{B C}\left([0,+\infty) ; \mathbf{R}^{n}\right)$.

- $B(X)$, the Banach space of bounded linear operators on the Banach space $X$ with norm $\|T\|:=\sup \{\|T x\|: x \in X,\|x\|=1\}$ for $T \in B(X)$.

The following lemma will be crucial in the proof of the main result of this paper.

Lemma $2.1([6])$ Let $E$ be a normed linear space. Let $S: E \rightarrow E$ be a completely continuous operator; that is, $S$ is continuous and the image of any bounded set is contained in a compact set. Let

$$
\zeta(S):=\{x \in E: x=\lambda S x \text { for some } 0<\lambda<1\} .
$$

Then either $\zeta(S)$ is unbounded or $S$ has a fixed point.

Lemma $2.2([4])$ Let $Q$ be a topological space. Let $S: \mathcal{B C}\left(Q ; \mathbf{R}^{n}\right) \rightarrow$ $\mathcal{B C}\left(Q ; \mathbf{R}^{n}\right)$ be a continuous operator. Suppose that for any bounded set $F \subset \mathcal{B C}\left(Q ; \mathbf{R}^{n}\right), S(F)$ is a bounded set and there exist $k$ bounded functions $\varphi_{j}: Q \rightarrow \mathbf{R}^{n_{j}}, j=1,2, \cdots, k$, such that

$$
|(S y)(t)-(S y)(s)| \leq \sum_{j=1}^{k}\left|\varphi_{j}(t)-\varphi_{j}(s)\right| \text { for all } t, s \in Q \text { and for all } y \in F
$$

Then $S$ is a completely continuous operator.

Moreover for the sake of completeness we recall the following result:

Lemma $2.3([2])$ Let $A:[0,+\infty) \rightarrow \mathcal{A}, t \mapsto A(t)$, be a bounded, integrable, continuous function. 
(i) The linear homogeneous differential system

$$
\dot{y}=A(t) y, \quad t \geq 0
$$

is uniformly stable; i.e., any solution of (4) is in $\mathcal{B C}\left([0,+\infty) ; \mathbf{R}^{n}\right)$ and is uniformly stable.

(ii) There exists a fundamental matrix $X(t)$ of solutions of (4) such that

$$
\left\|X(t) X^{-1}(s)\right\| \leq M_{2}, \quad 0 \leq s \leq t<+\infty
$$

for some constant $M_{2}>0$ and $E(t, s):=X(t) X^{-1}(s)$ is called the evolution operator of $A(t)$. One has

$$
\begin{gathered}
E(t, s)=I+\int_{s}^{t} A(\tau) E(\tau, s) d \tau \\
E(t, t)=I, \quad E(t, s) E(s, r)=E(t, r), \quad t, s, r \in[0,+\infty), \\
\frac{\partial E}{\partial t}=A(t) E(t, s), \quad \frac{\partial E}{\partial s}=-E(t, s) A(s), \quad \text { a.e. } t, s \in[0,+\infty) .
\end{gathered}
$$

\section{Main Result}

Let us consider the problem: Find an $x \in \mathcal{B C}\left([-r,+\infty) ; \mathbf{R}^{n}\right)$ such that

$$
\left\{\begin{array}{l}
\frac{d}{d t}\left[x(t)-g\left(t, x_{t}\right)\right]=A(t) x(t)+f\left(t, x_{t}, \int_{0}^{t} h\left(t, s, x_{s}\right) d s\right), \quad t \geq 0 \\
H(x)=L x
\end{array}\right.
$$

Theorem 3.1. Assume that the following hypotheses hold:

$\left(h_{1}\right) A:[0,+\infty) \rightarrow \mathcal{A}, t \mapsto A(t)$ is a bounded $\left(\|A(t)\| \leq M_{1}, \forall t \geq 0\right)$ integrable, continuous function for which

(i) there exists a continuous nondecreasing function $\gamma_{1}:[0,+\infty) \rightarrow$ $[0,+\infty)$ such that

$$
\left|\int_{0}^{t} X^{-1}(s) A(s) g\left(s, y_{s}\right) d s\right| \leq \gamma_{1}\left(\|y\|_{\infty}\right)
$$

(ii) there exists a continuous nondecreasing function $\gamma_{2}:[0,+\infty) \rightarrow$ $[0,+\infty)$ such that

$$
\left|\int_{0}^{t} X^{-1}(s) f\left(s, y_{s}, \int_{0}^{s} h\left(s, \tau, u_{\tau}\right) d \tau\right) d s\right| \leq \gamma_{2}\left(\|y\|_{\infty}\right) .
$$


$\left(h_{2}\right) g:[0,+\infty) \times \mathcal{C}\left([-r, 0] ; \mathbf{R}^{n}\right) \rightarrow \mathbf{R}^{n}$ is a continuous function such that

(i) $g\left(t, g_{t}\right)=: G_{y}(t)$ is a differentiable function for any $y \in \mathcal{B C}\left([-r,+\infty) ; \mathbf{R}^{n}\right)$;

(ii) $|g(t, \psi)| \leq c_{1}\|\psi\|_{\infty}+c_{2}$, with $c_{1}<1$.

(iii) $\left\|G_{y}(t)-G_{x}(t)\right\|_{\infty} \leq G\|x-y\|_{\infty}$ for a certain constant $G>0$ and for all $x, y \in \mathcal{C}\left([-r,+\infty) ; \mathbf{R}^{n}\right)$;

(iv) $\forall j, \exists \bar{G}_{j}:[0,+\infty) \rightarrow[0,+\infty)$, a bounded function such that

$$
\left|G_{y}\left(t_{1}\right)-G_{y}\left(t_{2}\right)\right| \leq\left|\bar{G}_{j}\left(t_{1}\right)-\bar{G}_{j}\left(t_{2}\right)\right| \quad \forall t_{1}, t_{2} \geq 0,\|y\|_{\infty} \leq j .
$$

$\left(h_{3}\right) f:[0,+\infty) \times \mathcal{C}\left([-r, 0] ; \mathbf{R}^{n}\right) \times \mathbf{R}^{n} \rightarrow X$ is a continuous function such that

(i) there exist an integrable function $p: \mathbf{R}^{+} \rightarrow \mathbf{R}^{+}$and a continuous nondecreasing function $\Omega:[0,+\infty) \rightarrow[0,+\infty)$ for which

$$
|f(t, x, y)| \leq p(t) \Omega\left(\|x\|_{\infty}+|y|\right), \quad t \geq 0, x \in \mathcal{C}\left([-r, 0] ; \mathbf{R}^{n}\right), y \in \mathbf{R}^{n}
$$

(ii) for every positive integer $j$ there exists an integrable function $\alpha_{j}$ : $[0,+\infty) \rightarrow[0,+\infty)$ such that

$$
\sup _{\|x\|_{\infty},|y| \leq j}|f(t, x, y)| \leq \alpha_{j}(t), \quad \text { for } t \geq 0 \text { a.e. }
$$

$\left(h_{4}\right) h:[0,+\infty) \times[0,+\infty) \times \mathcal{C}\left([-r, 0] ; \mathbf{R}^{n}\right)$ is a continuous function such that

(i) there exist an integrable function $m:[0,+\infty) \rightarrow[0,+\infty)$ and a continuous nondecreasing function $\Omega_{0}:[0,+\infty) \rightarrow[0,+\infty)$ for which

$$
|h(t, s, x)| \leq m(s) \Omega_{0}\left(\|x\|_{\infty}\right), \quad 0 \leq s \leq t<+\infty, x \in \mathcal{C}\left([-r, 0] ; \mathbf{R}^{n}\right),
$$

(ii)

$$
\int_{0}^{\infty} \frac{d s}{s+\Omega(s)+\Omega_{0}(s)}=+\infty
$$

$\left(h_{5}\right) L: \mathcal{B C}\left([-r,+\infty) ; \mathbf{R}^{n}\right) \rightarrow \mathcal{C}\left([-r, 0] ; \mathbf{R}^{n}\right)$ is a bounded linear operator such that

(i) $\|L\| \leq 1$,

(ii) If $u, v \in \mathcal{B C}\left([-r,+\infty) ; \mathbf{R}^{n}\right)$ are such that $\left.u\right|_{[-r, 0]}=\left.v\right|_{[-r, 0]}$, then $L u=L v$.

$\left(h_{6}\right) H: \mathcal{B C}\left([-r,+\infty) ; \mathbf{R}^{n}\right) \rightarrow \mathcal{C}\left([-r, 0] ; \mathbf{R}^{n}\right)$ is a continuous operator such that 
(i) $\|H u\|_{\infty} \leq M_{3}$ for a certain constant $M_{3}>1$.

$\left(h_{7}\right)$ There exists a linear continuous operator $\tilde{K}: \mathbf{R}^{n} \rightarrow \operatorname{ker} D$, where $D=$ $\frac{d}{d t}-A(t)$, such that

(i) $(\tilde{K} v)(0)=v$ for all $v \in \mathbf{R}^{n}$;

(ii) for every positive integer $j$ there exists a bounded function $V_{j}$ : $[0,+\infty) \rightarrow[0,+\infty)$ for which

$$
\mid\left(\tilde{K}((H(y))(0))\left(t_{1}\right)-\left(\tilde{K}((H(y))(0))\left(t_{2}\right)|\leq| V_{j}\left(t_{1}\right)-V_{j}\left(t_{2}\right) \mid\right.\right.
$$

for all $y \in \mathcal{B C}\left([-r,+\infty) ; \mathbf{R}^{n}\right)$ and $t_{1}, t_{2} \geq 0$;

(iii) define $K: \mathcal{C}\left([-r, 0] ; \mathbf{R}^{n}\right) \rightarrow \mathcal{B C}\left([-r, 0] ; \mathbf{R}^{n}\right)$ by

$$
(K u)(t):=\left\{\begin{array}{lc}
u(t) & -r \leq t \leq 0 \\
(\tilde{K}(u(0)))(t), & t \geq 0
\end{array}\right.
$$

(Note that $K$ is a bounded linear operator). We suppose that

$$
H(u)=L K H(u) \quad \text { for every } u \in \mathcal{B C}\left([-r,+\infty) ; \mathbf{R}^{n}\right) .
$$

Then problem $(\mathcal{P})$ has at least one solution.

Before proving Theorem 3.1, we first present an immediate consequence of it.

Corollary 3.2. Suppose that $\left(h_{1}\right)-\left(h_{4}\right)$ hold. Let $\varphi \in \mathcal{C}\left([-r, 0] ; \mathbf{R}^{n}\right)$. Then the Cauchy problem

$$
\left\{\begin{array}{l}
\frac{d}{d t}\left[x(t)-g\left(t, x_{t}\right)\right]=A(t) x(t)+f\left(t, x_{t}, \int_{0}^{t} h\left(t, s, x_{s}\right) d s\right), \quad t \geq 0 \\
\left.x\right|_{[-r, 0]}=\varphi
\end{array}\right.
$$

admits at least one solution.

Proof Corollary 3.2. If we define

$$
\begin{aligned}
L x & =\left.x\right|_{[-r, 0]}, & & x \in \mathcal{B C}\left([-r,+\infty) ; \mathbf{R}^{n}\right), \\
H x & =\varphi, & & x \in \mathcal{B C}\left([-r,+\infty) ; \mathbf{R}^{n}\right), \\
(\tilde{K} v)(t) & =E(t, 0) v, & & t \geq 0, v \in \mathbf{R}^{n},
\end{aligned}
$$

we see easily that $\left(h_{5}\right)-\left(h_{7}\right)$ are satisfied and therefore, the conclusion of Corollary 3.2 follows from Theorem 3.1. 
Remark. Corollary 3.2 extends, in $\mathbf{R}^{n}$, the main result of [3] in two ways: the time-dependence of the operator $A(t)$ and the unboundedness of the interval $[0,+\infty)$.

Proof of Theorem 3.1. First we extend to $[-r, 0]$ the functions useful to us, putting, for $u \in \mathcal{B C}\left([-r, 0) ; \mathbf{R}^{n}\right)$ :

$$
\begin{gathered}
\bar{g}\left(t, u_{t}\right)=\left\{\begin{array}{l}
g\left(t, u_{t}\right), \\
g\left(0, u_{0}\right), \quad-r \leq t \leq 0,
\end{array}\right. \\
\bar{E}(t, 0) g\left(0, u_{0}\right)= \begin{cases}E(t, 0) g\left(0, u_{0}\right), & t \geq 0, \\
g\left(0, u_{0}\right), & -r \leq t \leq 0,\end{cases} \\
w_{u}(t)= \begin{cases}\int_{0}^{t} E(t, s) A(s) g\left(s, u_{s}\right) d s, & t \geq 0, \\
0, & t \leq 0,\end{cases} \\
z_{u}(t)= \begin{cases}\int_{0}^{t} E(t, s) f\left(s, u_{s}, \int_{0}^{s} h\left(s, \tau, u_{\tau}\right) d \tau\right) d s, & t \geq 0, \\
0, & t \leq 0 .\end{cases}
\end{gathered}
$$

Now we consider the operator $S: \mathcal{B C}\left([-r,+\infty) ; \mathbf{R}^{n}\right) \rightarrow \mathcal{B C}\left([-r,+\infty) ; \mathbf{R}^{n}\right)$ defined by

$$
S(u)=K(H(u))+\bar{g}\left(\cdot, u_{.}\right)-\bar{E}(\cdot, 0) g\left(0, u_{0}\right)+w_{u}+z_{u} .
$$

We will show that the fixed points of $S$ are solutions of problem $(\mathcal{P})$ and that $S$ is a continuous compact operator for which the set $\zeta(S)$ is bounded. This is sufficient, by Lemma 2.1, to conclude that $S$ has fixed points.

Step 1: The fixed points of $S$ are solutions of problem $(\mathcal{P})$.

Proof of Step 1. Let $u \in \mathcal{B C}\left([-r,+\infty) ; \mathbf{R}^{n}\right)$. Then, by the linearity of $L$,

$$
L(S u)=L K H u+L(\bar{y}(\cdot, u .))-L\left(\bar{E}(\cdot, 0) g\left(0, u_{0}\right)\right)+L\left(w_{u}\right)+L\left(z_{u}\right) .
$$

Moreover, by $\left(h_{5}\right)(\mathrm{ii}), L\left(w_{u}\right)=L\left(z_{u}\right)=0$ since $\left.w_{u}\right|_{[-r, 0]}=\left.z_{u}\right|_{[-r, 0]}=0$, and $L(\bar{g}(\cdot, u))=.L\left(\bar{E}(\cdot, 0) g\left(0, u_{0}\right)\right)$ since $\left.\bar{g}(\cdot, u)\right|_{.[-r, 0]}=\left.\bar{E}(\cdot, 0) g\left(0, u_{0}\right)\right|_{[-r, 0]}$. So

$$
L S u=L K H u=H u \quad\left(\text { by }\left(h_{7}\right)(\mathrm{iii})\right) .
$$

In addition, we have by $\left(h_{7}\right)($ iii) and (8) that, for $t \geq 0$,

$$
\begin{aligned}
\frac{d}{d t}(S(u))(t)= & \frac{d}{d t}\left[(K(H(u)))(t)+g\left(t, u_{t}\right)-E(t, 0) g\left(0, u_{0}\right)+w_{u}(t)+z_{u}(t)\right] \\
= & \frac{d}{d t}(\tilde{K}(H(u)(0)))(t)+\frac{d}{d t} g\left(t, u_{t}\right)-A(t) E(t, 0) g\left(0, u_{0}\right) \\
& +A(t) g\left(t, u_{t}\right)+A(t) w_{u}(t)+f\left(t, u_{t}, \int_{0}^{t} h\left(t, s, u_{s}\right) d s\right)+A(t) z_{u}(t) .
\end{aligned}
$$


Since the range of $\tilde{K}$ is contained in $\operatorname{ker} D\left(\right.$ see $\left.\left(h_{7}\right)\right)$, we have

$$
\frac{d}{d t}(\tilde{K}(H(u)(0)))(t)=A(t)(\tilde{K}(H(u)(0)))(t)=A(t)(K H u)(t),
$$

in such a way that, for $t \geq 0$,

$$
\frac{d}{d t}\left[(S(u))(t)-g\left(t, u_{t}\right)\right]=A(t)(S(u))(t)+f\left(t, u_{t}, \int_{0}^{t} h\left(t, s, u_{s}\right) d s\right) .
$$

So, if $x$ is a fixed point of $S, x=S x,(10)$ and $(9)$ ensure that problem $(\mathcal{P})$ is solved by such a fixed point.

Step 2: $S$ is a continuous operator.

Proof of Step 2. Let $\left\{u_{m}\right\} \subset \mathcal{B C}\left([-r,+\infty) ; \mathbf{R}^{n}\right)$ be such that $u_{m} \stackrel{\|\cdot\|_{\infty}}{\longrightarrow} u$. We show that $S\left(u_{m}\right) \stackrel{\|\cdot\|_{\infty}}{\longrightarrow} S(u)$. By definition of $S$, it is sufficient to show that

$$
\begin{gathered}
K\left(H\left(u_{m}\right)\right) \stackrel{\|\cdot\|_{\infty}}{\longrightarrow} K(H(u)), \\
\bar{g}\left(\cdot,\left(u_{m}\right) .\right) \stackrel{\|\cdot\|_{\infty}}{\longrightarrow} \bar{g}(\cdot, u .), \\
\bar{E}(\cdot, 0) g\left(0,\left(u_{m}\right)_{0}\right) \stackrel{\|\cdot\|_{\infty}}{\longrightarrow} \bar{E}(\cdot, 0) g\left(0, u_{0}\right), \\
w_{u_{m}} \stackrel{\|\cdot\|_{\infty}}{\longrightarrow} w_{u}, \\
z_{u_{m}} \stackrel{\|\cdot\|_{\infty}}{\longrightarrow} z_{u} .
\end{gathered}
$$

Now, (11) is an immediate consequence of the continuity of the operators $K$ and $H$. To obtain $(12)$, we note, by $\left(h_{2}\right)$ (iii), that

$$
\left\|\bar{g}\left(t,\left(u_{m}\right)_{t}\right)-\bar{g}\left(t, u_{t}\right)\right\| \leq G\left\|u_{m}-u\right\|_{\infty} \rightarrow 0 .
$$

To obtain (13), we note that

$$
\begin{aligned}
& \left\|\bar{E}(\cdot, 0) g\left(0,\left(u_{m}\right)_{0}\right)-\bar{E}(\cdot, 0) g\left(0, u_{0}\right)\right\|_{\infty} \\
= & \left\|E(t, 0)\left(g\left(0,\left(u_{m}\right)_{0}\right)-g\left(0, u_{0}\right)\right)\right\| \quad(\text { by Lemma } 2.3(\mathrm{ii})) \\
\leq & M_{2}\left|g\left(0,\left(u_{m}\right)_{0}\right)-g\left(0, u_{0}\right)\right| \rightarrow 0 \quad \text { by }\left(h_{2}\right) .
\end{aligned}
$$

To obtain (14), using Lemma 2.3(iii) and $\left(h_{2}\right)$ (ii) we note that

$$
\begin{aligned}
\left\|w_{u_{m}}-w_{u}\right\|_{\infty} & =\sup _{-r \leq t<\infty}\left|w_{u_{m}}(t)-w_{u}(t)\right| \\
& =\sup _{0 \leq t<\infty}\left|w_{u_{m}}(t)-w_{u}(t)\right| \\
& =\left\|\int_{0}^{t} E(t, s) A(s)\left[g\left(s,\left(u_{m}\right)_{s}\right)-g\left(s, u_{s}\right)\right] d s\right\|_{\infty} \\
& \leq M_{2} G\left\|u_{m}-u\right\|_{\infty} \cdot \int_{0}^{\infty}\|A(s)\| d s \rightarrow 0 \quad \text { by }\left(h_{1}\right) .
\end{aligned}
$$


Finally, to obtain (15), we note first that by the continuity of $f$ and $g$, it follows that

$$
\psi_{m}(s)=f\left(s,\left(u_{m}\right)_{s}, \int_{0}^{s} h\left(s, \tau,\left(u_{m}\right)_{\tau}\right) d \tau\right)-f\left(s, u_{s}, \int_{0}^{s} h\left(s, \tau, u_{\tau}\right) d \tau\right) \rightarrow 0
$$

pointwise. Moreover, since $\left\|u_{m}-u\right\|_{\infty} \rightarrow 0$, there exists $j \in \mathbf{N}$ such that $\left\|u_{m}\right\|_{\infty},\|u\|_{\infty} \leq j$. So, by $\left(h_{4}\right)(\mathrm{i})$

$$
\begin{aligned}
\left|\int_{0}^{s} h\left(s, \tau,\left(u_{m}\right)_{\tau}\right) d \tau\right| & \leq \int_{0}^{s} m(s) \Omega_{0}\left(\left\|u_{m}\right\|_{\infty}\right) d s \\
& \leq \Omega_{0}(j) \int_{0}^{s} m(s) d s \\
\left|\int_{0}^{s} h\left(s, \tau, u_{\tau}\right) d \tau\right| & \leq \Omega_{0}(j) \int_{0}^{s} m(s) d s .
\end{aligned}
$$

Put

$$
j^{\prime}=\max \left\{j,\left[\Omega_{0}(j) \int_{0}^{s} m(s) d s\right]+1\right\},
$$

where we use $[r]$ to denote the integer part of a positive real number $r$. Then by $\left(h_{3}\right)($ ii) we see that

$$
\begin{aligned}
\left|\psi_{m}(s)\right| & \leq\left|f\left(s,\left(u_{m}\right)_{s}, \int_{0}^{s} h\left(s, \tau,\left(u_{m}\right)_{\tau}\right) d \tau\right)\right|+\left|f\left(s, u_{s}, \int_{0}^{s} h\left(s, \tau, u_{\tau}\right) d \tau\right)\right| \\
& \leq 2 \alpha_{j^{\prime}}(s)
\end{aligned}
$$

i.e., $\psi_{m}(s)$ converges pointwise to 0 and it is dominated by an integrable function. Hence by Lemma 2.3(ii),

$$
\begin{aligned}
\left\|z_{u_{m}}-z_{u}\right\|_{\infty} & =\sup _{-r \leq t<\infty}\left|z_{u_{m}}(t)-z_{u}(t)\right| \\
& =\sup _{0 \leq t<\infty}\left|z_{u_{m}}(t)-z_{u}(t)\right| \\
& =\left\|\int_{0}^{t} E(t, s) \psi_{m}(s) d s\right\|_{\infty} \\
& \leq M_{3} \int_{0}^{\infty}\left|\psi_{m}(s)\right| d s \longrightarrow 0
\end{aligned}
$$

by the dominated convergence theorem.

Step 3: $S$ is a compact operator.

Proof of Step 3. Let

$$
B_{j}=\left\{u \in \mathcal{B C}\left([-r,+\infty) ; \mathbf{R}^{n}\right):\|u\|_{\infty} \leq j\right\} .
$$

Thanks to Lemma 2.2, it is enough to show that $S\left(B_{j}\right)$ is a bounded set and that it is possible to control the oscillations of each function in $S\left(B_{j}\right)$ by means 
of a finite number of bounded functions. The boundedness of $S\left(B_{j}\right)$ follows from the inequalities:

$$
\begin{aligned}
\|K(H(u))\|_{\infty} & \leq\|K\| H u\left\|_{\infty} \leq M_{3}\right\| K \| \quad\left(\text { by }\left(h_{6}\right)(\mathrm{i})\right) \\
\left|\bar{g}\left(t, u_{t}\right)\right| & \leq c_{1 j}+C_{2} \quad\left(\text { by }\left(h_{2}\right)(\mathrm{ii})\right) \\
\left|\bar{E}(t, 0) g\left(0, u_{0}\right)\right| & \leq M_{2}\left(c_{1 j}+C_{2}\right) \quad\left(\text { by Lemma 2.3(ii) and }\left(h_{2}\right)(\mathrm{ii})\right) \\
\left\|w_{u}\right\|_{\infty} & =\left\|\int_{0}^{t} E(t, s) A(s) g\left(s, u_{s}\right) d s\right\|_{\infty} \\
& \leq M_{2}\left(c_{1 j}+C_{2}\right) \int_{0}^{\infty}\|A(s)\| d s\left(\text { by Lemma } 2.3(\mathrm{ii}),\left(h_{2}\right)(\mathrm{ii}),\left(h_{1}\right)\right) \\
\left\|z_{u}\right\|_{\infty} & \left.=\| \int_{0}^{t} E(t, s) f\left(s, u_{s}, \int_{0}^{s} h\left(s, \tau, u_{\tau}\right) d \tau\right)\right) d s \|_{\infty} \\
& \leq M_{2} \int_{0}^{\infty} \alpha_{j}(t) d t \quad\left(\text { by Lemma 2.3(ii), }\left(h_{3}\right)(\mathrm{ii}) \text { and }\left(h_{4}\right)\right) .
\end{aligned}
$$

To control the oscillations of $S\left(B_{j}\right)$ we need to distinguish three cases: $0 \leq t_{1}<$ $t_{2}, t_{1}<0<t_{2}$ and $t_{1}<t_{2} \leq 0$. We consider here only the case $0 \leq t_{1}<t_{2}$, the others being similar.

Let us assume $0 \leq t_{1}<t_{2}$ and $u \in B_{j}$. Then

$$
\begin{gathered}
\left|(S(u))\left(t_{1}\right)-(S(u))\left(t_{2}\right)\right|=\mid(K(H(u)))\left(t_{1}\right)+g\left(t_{1}, u_{t_{1}}\right)-E\left(t_{1}, 0\right) g\left(0, u_{0}\right) \\
\quad+\int_{0}^{t_{1}} E\left(t_{1}, s\right) A(s) g\left(s, u_{s}\right) d s \\
\left.+\int_{0}^{t_{1}} E\left(t_{1}, s\right) f\left(s, u_{s}, \int_{0}^{s} h\left(s, \tau, u_{\tau}\right) d \tau\right)\right) d s \\
-(K(H(u)))\left(t_{2}\right)-g\left(t_{2}, u_{t_{2}}\right)+E\left(t_{2}, 0\right) g\left(0, u_{0}\right) \\
\quad-\int_{0}^{t_{2}} E\left(t_{2}, s\right) A(s) g\left(s, u_{s}\right) d s \\
\left.-\int_{0}^{t_{2}} E\left(t_{2}, s\right) f\left(s, u_{s}, \int_{0}^{s} h\left(s, \tau, u_{\tau}\right) d \tau\right)\right) d s \mid \\
\left.\leq \mid \tilde{K}(H(u)(0)))\left(t_{1}\right)-\tilde{K}(H(u)(0))\right)\left(t_{2}\right) \mid \\
+\left|g\left(t_{1}, u_{t_{1}}\right)-g\left(t_{2}, u_{t_{2}}\right)\right|+\left|\left(E\left(t_{1}, 0\right)-E\left(t_{2}, 0\right)\right) g\left(0, u_{0}\right)\right| \\
+\left|\int_{0}^{t_{1}}\left(E\left(t_{1}, s\right)-E\left(t_{2}, s\right)\right) A(s) g\left(s, u_{s}\right) d s\right| \\
+\left|\int_{t_{1}}^{t_{2}} E\left(t_{2}, s\right) A(s) g\left(s, u_{s}\right) d s\right| \\
\left.+\mid \int_{0}^{t_{1}}\left(E\left(t_{1}, s\right)-E\left(t_{2}, s\right)\right) f\left(s, u_{s}, \int_{0}^{s} h\left(s, \tau, u_{\tau}\right) d \tau\right)\right) d s \mid
\end{gathered}
$$




$$
\left.+\mid \int_{t_{1}}^{t_{2}} E\left(t_{2}, s\right) f\left(s, u_{s}, \int_{0}^{s} h\left(s, \tau, u_{\tau}\right) d \tau\right)\right) d s \mid
$$

Now

$$
\begin{gathered}
\left.\mid \tilde{K}(H(u)(0)))\left(t_{1}\right)-\tilde{K}(H(u)(0))\right)\left(t_{2}\right)|\leq| V_{j}\left(t_{1}\right)-V_{j}\left(t_{2}\right) \mid, \quad\left(\text { by }\left(h_{7}\right)(\mathrm{ii})\right) \\
\left|g\left(t_{1}, u_{t_{1}}\right)-g\left(t_{2}, u_{t_{2}}\right)\right| \leq\left|\bar{G}_{j}\left(t_{1}\right)-\bar{G}_{j}\left(t_{2}\right)\right|, \quad\left(\text { by }\left(h_{2}\right)(\mathrm{iv})\right) \\
\left|\left(E\left(t_{1}, 0\right)-E\left(t_{2}, 0\right)\right) g\left(0, u_{0}\right)\right| \leq\left\|E\left(t_{1}, 0\right)-E\left(t_{2}, 0\right)\right\|\left(c_{1 j}+C_{2}\right), \\
\left|\int_{0}^{t_{1}}\left(E\left(t_{1}, s\right)-E\left(t_{2}, s\right)\right) A(s) g\left(s, u_{s}\right) d s\right| \leq\left\|X\left(t_{1}\right)-X\left(t_{2}\right)\right\| \gamma_{1}(j), \quad\left(\text { by }\left(h_{1}\right)(\mathrm{i})\right) \\
\left|\int_{t_{1}}^{t_{2}} E\left(t_{2}, s\right) A(s) g\left(s, u_{s}\right) d s\right| \leq M_{2}\left(c_{1 j}+C_{2}\right)\left(\int_{0}^{t_{1}}\|A(s)\| d s-\int_{0}^{t_{1}}\|A(s)\| d s\right) \\
\quad\left(\text { by Lemma } 2.3(\mathrm{ii}),\left(h_{2}\right)(\mathrm{ii}), \text { and }\left(h_{1}\right)\right) \\
\left.\mid \int_{0}^{t_{1}}\left(E\left(t_{1}, s\right)-E\left(t_{2}, s\right)\right) f\left(s, u_{s}, \int_{0}^{s} h\left(s, \tau, u_{\tau}\right) d \tau\right)\right) d s \mid \leq \quad\left\|X\left(t_{1}\right)-X\left(t_{2}\right)\right\| \gamma_{2}(j) \\
\quad\left(\text { by }\left(h_{1}\right)(\mathrm{ii})\right)
\end{gathered}
$$

and by Lemma 2.3(ii), $\left(h_{3}\right)(\mathrm{ii})$ and $\left(h_{4}\right)(\mathrm{i})$,

$$
\left.\mid \int_{t_{1}}^{t_{2}} E\left(t_{2}, s\right) f\left(s, u_{s}, \int_{0}^{s} h\left(s, \tau, u_{\tau}\right) d \tau\right)\right) d s \mid \leq M_{2}\left(\int_{0}^{t_{2}} \alpha_{j^{\prime}}(s) d s-\int_{0}^{t_{1}} \alpha_{j^{\prime}}(s) d s\right) .
$$

So, defining

$$
\begin{gathered}
\varphi_{1}(t)=V_{1}(t), \quad \varphi_{2}(t)=\bar{G}_{j}(t), \quad \varphi_{3}(t)=\left(c_{1 j}+C_{2}\right) E(t, 0), \quad \varphi_{4}(t)=\gamma_{1}(j) X(t), \\
\varphi_{5}(t)=\int_{0}^{t}\|A(s)\| d s, \quad \varphi_{6}(t)=\gamma_{2}(j) X(t), \quad \varphi_{7}(t)=\int_{0}^{t}\left\|\alpha_{j^{\prime}}(s)\right\| d s,
\end{gathered}
$$

we obtain that

$$
\left|(S(u))\left(t_{1}\right)-(S(u))\left(t_{2}\right)\right| \leq \sum_{k=1}^{7}\left|\varphi_{k}\left(t_{1}\right)-\varphi_{k}\left(t_{2}\right)\right| \quad \text { for all } u \in B_{j}
$$

in such a way that the thesis follows from Lemma 2.2.

Step 4. The set $\zeta(S)$ is bounded.

Proof of Step 4. This follows essentially from the same idea as in [3]. First of all, if $-r \leq t<0$, then $(S(u))(t)=(H u)(t)$. So, by $\left(h_{6}\right)(\mathrm{i})$, it follows that 
$|(S(u))(t)| \leq M_{3}$ for each $u \in \mathcal{B C}\left([-r,+\infty) ; \mathbf{R}^{n}\right)$. Hence we only consider $t \geq 0$. Let $u(t)=\lambda(S(u))(t)$. Thus

$$
\begin{aligned}
|u(t)|= & |\lambda||(S(u))(t)| \leq|(S(u))(t)| \\
\leq \quad & |(K(H(u)))(t)|+\left|g\left(t, u_{t}\right)\right|+\left|E(t, 0) g\left(0, u_{0}\right)\right| \\
& +\left|\int_{0}^{t} E(t, s) A(s) g\left(s, u_{s}\right) d s\right| \\
& +\left|\int_{0}^{t} E(t, s) f\left(s, u_{s}, \int_{0}^{s} h\left(s, \tau, u_{\tau}\right) d \tau\right) d s\right| \\
\leq \quad & \|K\| M_{3}+c_{1}\left\|u_{t}\right\|_{\infty}+c_{2}(t)+M_{2}\left(c_{1}\left\|u_{0}\right\|_{\infty}+c_{2}(0)\right) \\
& +M_{2} \int_{0}^{t}\left(c_{1}\left\|u_{s}\right\|_{\infty}+c_{2}(s)\right)\|A(s)\| d s \\
& +M_{2} \int_{0}^{t} p(s) \Omega\left(\left\|u_{s}\right\|_{\infty}+\left|\int_{0}^{s} m(\tau) \Omega_{0}\left(\left\|u_{\tau}\right\|_{\infty}\right) d \tau\right|\right) d s .
\end{aligned}
$$

But,

$$
\left\|u_{0}\right\|_{\infty}=\sup _{-r \leq \theta \leq 0}|u(\theta)| \text { and }|u(\theta)|=|\lambda||(S(u))(t)| \leq M_{3},
$$

it follows that

$$
\begin{aligned}
|u(t)| \leq & \|K\| M_{3}+c_{1}\left\|u_{t}\right\|_{\infty}+c_{2}(t)+M_{2}\left(c_{1} M_{3}+c_{2}(0)\right) \\
& +M_{2} \int_{0}^{t}\left(c_{1}\left\|u_{s}\right\|_{\infty}+c_{2}(s)\right)\|A(s)\| d s \\
& +M_{2} \int_{0}^{t} p(s) \Omega\left(\left\|u_{s}\right\|_{\infty}+\left|\int_{0}^{s} m(\tau) \Omega_{0}\left(\left\|u_{\tau}\right\|_{\infty}\right) d \tau\right|\right) d s .
\end{aligned}
$$

Consider the function $\mu:[0,+\infty) \rightarrow[0,+\infty)$ defined by

$$
\mu(t):=\max \{|u(s)|:-r \leq s \leq t\} .
$$

Let $t^{*} \in[-r, t]$ be such that

$$
\mu(t)=\left|u\left(t^{*}\right)\right|
$$

If $t^{*}<0$, then $\mu(t)=\left|u\left(t^{*}\right)\right| \leq M_{3}$. If $t^{*} \geq 0$, then from

$$
\left\|u_{t}\right\|_{\infty}=\sup _{-r \leq \theta \leq 0}|u(t+\theta)|=\sup _{-r+t \leq \xi \leq t}|u(\xi)| \leq \sup _{-r \leq \xi \leq t}|u(\xi)|=\mu(t),
$$

the previous inequality yields

$$
\begin{aligned}
\mu(t) \leq & \|K\| M_{3}+c_{1} \mu(t)+c_{2}+M_{2}\left(c_{1} M_{3}+c_{2}\right) \\
& +M_{2} c_{1} \int_{0}^{t} \mu(s)\|A(s)\| d s+M_{2} c_{2} \int_{0}^{\infty}\|A(s)\| d s \\
& +M_{2} \int_{0}^{t} p(s) \Omega\left(\mu(s)+\int_{0}^{s} m(\tau) \Omega_{0}(\mu(\tau)) d \tau\right) d s .
\end{aligned}
$$


This implies that

$$
\begin{aligned}
\mu(t) \leq & \frac{1}{1-c_{1}}\left[\|K\| M_{3}+c_{2}+M_{2}\left(c_{1} M_{3}+c_{2}\right)\right. \\
& +M_{2} c_{2} \int_{0}^{\infty}\|A(s)\| d s+M_{2} c_{1} \int_{0}^{t} \mu(s)\|A(s)\| d s \\
& \left.+M_{2} \int_{0}^{t} p(s) \Omega\left(\mu(s)+\int_{0}^{s} m(\tau) \Omega_{0}(\mu(\tau)) d \tau\right) d s\right] .
\end{aligned}
$$

Denoting by $v(t)$ the right-hand side of the last inequality, we have

$$
\begin{aligned}
c:=v(0) & =\frac{1}{1-c_{1}}\left[\|K\| M_{3}+c_{2}+M_{2}\left(c_{1} M_{3}+c_{2}\right)+M_{2} c_{2} \int_{0}^{\infty}\|A(s)\| d s\right], \\
\mu(t) & \leq v(t) \text { for } t \geq 0, \\
v^{\prime}(t) & =\frac{M_{2} c_{1}}{1-c_{1}} \mu(t)\|A(t)\|+\frac{M_{2}}{1-c_{1}} p(t) \Omega\left(\mu(t)+\int_{0}^{t} m(s) \Omega_{0}(\mu(s)) d s\right) \\
& \leq \frac{M_{2} c_{1}}{1-c_{1}} v(t)\|A(t)\|+\frac{M_{2}}{1-c_{1}} p(t) \Omega\left(v(t)+\int_{0}^{t} m(s) \Omega_{0}(v(s)) d s\right) .
\end{aligned}
$$

Putting

$$
w(t)=v(t)+\int_{0}^{t} m(s) \Omega_{0}(v(s)) d s
$$

we get

$$
\begin{aligned}
w(0) & =v(0)=c \\
v(t) & \leq w(t) \\
w^{\prime}(t) & =v^{\prime}(t)+m(t) \Omega_{0}(v(t)) \\
& \leq \frac{M_{2} c_{1}}{1-c_{1}} v(t)\|A(t)\|+\frac{M_{2}}{1-c_{1}} p(t) \Omega(w(t))+m(t) \Omega_{0}(v(t)) \\
& \leq \frac{M_{2} c_{1}}{1-c_{1}}\|A(t)\| w(t)+\frac{M_{2}}{1-c_{1}} p(t) \Omega(w(t))+m(t) \Omega_{0}(w(t)) .
\end{aligned}
$$

Let now

$$
\xi(t):=\max \left\{\frac{M_{2} c_{1}}{1-c_{1}}\|A(t)\|, \frac{M_{2}}{1-c_{1}} p(t), m(t)\right\} .
$$

Then from the previous inequality we have

$$
w^{\prime}(t) \leq \xi(t)\left[w(t)+\Omega(w(t))+\Omega_{0}(w(t))\right] .
$$

This implies that

$$
\frac{w^{\prime}(t)}{w(t)+\Omega(w(t))+\Omega_{0}(w(t))} \leq \xi(t)
$$

and so, for any $b>0$,

$$
\int_{0}^{b} \frac{w^{\prime}(t)}{w(t)+\Omega(w(t))+\Omega_{0}(w(t))} d t \leq \int_{0}^{b} \xi(t) d t \leq \int_{0}^{\infty} \xi(t) d t=: \Gamma<\infty
$$


that is,

$$
\int_{w(0)}^{w(b)} \frac{d s}{s+\Omega_{0}(s)+\Omega(s)} d s \leq \Gamma .
$$

This, together with hypothesis $\left(h_{4}\right)$ (ii), permits us to conclude that $w(t)$ is bounded by a constant $\Lambda$, say, depending on the functions $\Omega, \Omega_{0}, A, p$, and $m$. Summarizing, $u \in \zeta(S)$ implies that $\|u\|_{\infty} \leq\|\mu\|_{\infty} \leq\|v\|_{\infty} \leq\|w\|_{\infty} \leq \Lambda$.

\section{References}

[1] M. Benchohra, J. Henderson, and S. K. Ntouyas, Existence results for impulsive multivalued semilinear neutral functional differential inclusions in Banach spaces, J. Math. Anal. Appl. 263 (2001), 763-780.

[2] R. Conti, Linear Differential Equations, Academic Press, London, 1976.

[3] J. P. Dauer and K. Balachandran, Existence of solutions of nonlinear neutral integrodifferential equations in Banach spaces, J. Math. Anal. Appl. 251 (2000), 93-105.

[4] E. De Pascale, G. Lewicki, and G. Marino, Some conditions for compactness in $B C(Q)$ and their application to boundary value problems, Analysis 22 (2002), 21-32.

[5] E. Hernandez and H. R. Hernandez, Existence results for partial neutral functional differential equations with unbounded delay, J. Math. Anal. Appl. 221 (1998), 452-475.

[6] H. Schaefer, Über die methode der a priori schranken, Math. Ann. 129 (1955), 415-416.

[7] C. C. Travis and G. F. Webb, Existence and stability for partial functional integrodifferential equations, Trans. Amer. Math. Soc. 200 (1974), 395418.

Received: October 10, 2005 